\title{
A Real-Time Urban Traffic Detection Algorithm Based on Spatio-Temporal OD Matrix in Vehicular Sensor Network*
}

\author{
Ke Zhang, Guangtao Xue \\ Department of Compute Science \& Technology, Shanghai Jiao Tong University, Shanghai, China \\ E-mail: xue-gt@cs.sjtu.edu.cn \\ Received July 4, 2010; revised August 7, 2010; accepted September 10, 2010
}

\begin{abstract}
Currently, there are kinds of algorithms in order to detect real-time urban traffic condition. Most of these approaches consider speed of vehicles as a main metric to describe traffic situation. In this paper, we find out two important observations through several experiments. (1) In urban city, the speed of vehicles is influenced significantly by some factors such as traffic lights delay and road condition. The actual situation rarely satisfy hypothesis required for these solutions. Therefore, these traditional algorithms do not work well in practical environment. (2) Traffic volume on a road segment shows strong pattern and changes smoothly at adjacent time. This feature of traffic volume inspires us to define a metric: traffic-rate, which is used to detect traffic condition in real time. In our solution, we develop a novel traffic-detection algorithm based on originaldestination (OD) matrix. We illustrate our approach and measure its performance in real environment. The performance evaluations confirm the effectiveness of our algorithm.
\end{abstract}

Keywords: KNN; Linear Least Square; OD Matrix; Traffic Status Detection

\section{Introduction}

Nowadays, traffic congestion has been a serious problem in many urban cities. In China, it caused about 5\%-8\% GDP wasted each year. Therefore, governments cost million dollars to build Intelligent Transportation System (ITS). In response to these challenges, OriginalDestination (OD) matrix is often required as application input to provide service for transportation. OD matrix is a non-negative matrix $f(i, j)$ which represents volumes of traffic from a source $i$ to a destination $j$. In a road network, element $f(i, j)$ in the OD matrix means the number of vehicles from road segment $i$ to road segment $j$.However, traditional algorithms to construct OD matrix do not work well in real world, since their solutions do not consider features of real traffic condition. Furthermore, the traditional algorithms of traffic detection have limitations when runs in real environment. In their solutions, speed of vehicles is considered as a main metric to identify traffic states. Nevertheless, we find that speed is influenced significantly by traffic lights.

\footnotetext{
*Supported by the National Natural Science Foundation of China under Grant No. 60970106, the National Grand Fundamental Research 973 Program of China under Grant No.2006CB303000, and Key Program of National MIIT under Grant 2009ZX03006-004.
}

In this paper, we study this problem using GPS devices which have been equipped in taxis and buses in Shanghai. All GPS vehicular data are transferred to the local APs which are deployed in intersections, and GPS reports would be sent to a data center, where we run our algorithm to construct OD matrix and analyze traffic condition. We receive a great support from Shanghai government and have deployed a cost-effective system to collect traffic information. However, there are still lots of challenges to process information in a metropolis like Shanghai.

First of all, due to vehicular GPS signal is always varying, all GPS location data we collected are not only large and noisy but not uniformly distribute. Some areas near downtown produce most of traffic volumes. Figure 1 shows millions of GPS reports on each road from January 5 to January 20 in 2007. In addition, according to our statistics, almost 30\% vehicles lost their GPS reports. Besides, the solution must consider the fact that not every vehicle has installed GPS device.

Although taxies and buses have installed vehicular wireless communication devices whose communication range is about 200 meters, it is costly if we deploy AP every 200 meter. Thus, we need to interpolate traffic volumes on road segments that do not have AP. Fur 


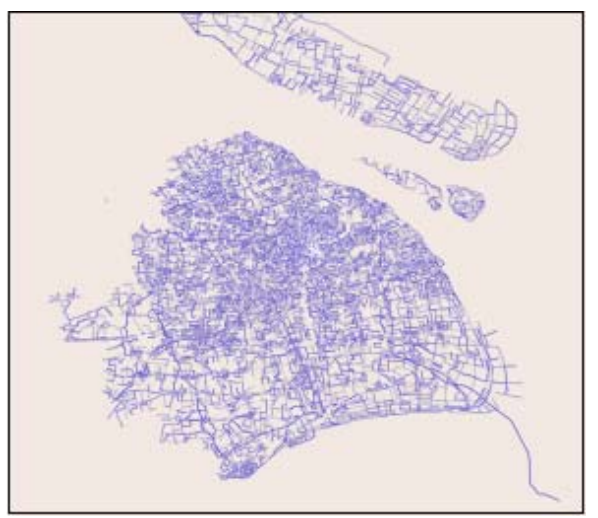

Figure 1. GIS map of Shanghai with spatial distribution of GPS reports from Jan.5 to Jan.20, 2007.

thermore, because of errors of GPS data (the range of the error is about 20 meters to 100 meters), we have to firstly apply our own GPS-correcting algorithms to abandon "dirty" data and amend incorrect ones.

The last but not the least, it is difficult to capture every factor which has effect on traffic condition. For instance, traffic lights delay and complicated condition of road have significant impact on traffic condition. Meanwhile, these factors are difficult to be estimated using a simple model. This is why most proposed algorithms do not work well in real life.

Fortunately, we find out a spatial-temporal model that is able to overcome these limitations to reconstruct OD matrix. In addition, we define a metric: Traffic-Rate, which is used to describe current traffic condition. We are going to discuss them in Subsection 2.5.

The remainder of this paper is organized as follows: Subsection 1.1 provides background and compares our approach with related works. We then introduce our spatial-temporal algorithm and traffic-detection solution in detail in Section 2. We demonstrate our evaluation result in Section 3. Finally, we present conclusion in Section 4.

\subsection{Related Work}

Due to importance of OD matrix, there are a number of works focus on how to estimate OD matrix accurately in recent years [1-3]. However, most of their solutions work on highways or freeways, where traffic lights timing and behavior of drivers are not issues because there are no intersections and vehicles' routes followed a certain way. On the other hand, traffic situation of an urban is so complicate that the technical conditions which required for these solutions are difficult to be satisfied. Yin Zhang et al [4] illustrate a novel algorithm to estimate a traffic matrix in Internet whereas our solution runs in the $\mathbf{V e}$ hicular Sensor Network (VSN), more importantly, they do not give the fact to prove their observation.
With rapid evolvement of intelligent traffic system (ITS), a lot of efforts focus on monitoring traffic and incidents detections installing sensors like camera on roads [5]. From a practical perspective, these approaches need large sums of money to deploy numbers of sensors. Moreover, Lin et al [6] and Coifman et al [7] propose schemes to detect traffic condition by installing traffic detectors. Meanwhile, their solutions work on freeway not an urban area. How to define an appropriate metric to describe traffic condition is also a hot topic for researchers. For example, Jungkeun Yoon et al [8] analyzed main factors which influence traffic states. Furthermore, some researchers [9-11] propose interesting schemes to estimate velocity of vehicles to reflect traffic condition. However, they do not take condition of roads into account. Therefore, their solutions have limitations when runs in real environment.

\section{Spatio-Temporal Original-Destination Matrix}

In this section, we give a brief introduction to OD matrix first. Then we present our algorithm in detail. This construction algorithm consists of two important rules that are observed by experiments. We take advantage of OD matrix to implement traffic-detection solution.

\subsection{Original-Destination Matrix}

For a road network with $N$ intersections which connect $M$ road segments, the OD matrix is a square $M \times M$ matrix. In this paper, the element $f(i, j, t)$ in OD matrix represents traffic volumes from roadi $_{i}$ to road $_{i}$ during $[t, t+\Delta t)$. In order to get more convenience for calculation, we convert OD matrix as a 2-dimensional array, where columns represent OD matrix at different time while the rows represent the evolution of traffic volumes in each route.

\subsection{The Spatial Component}

After extracting and analyzing millions of real vehicles GPS reports, we find the first important fact that there are strong correlations of traffic volumes among road segments. In practice, road $i$ to some extent has similar traffic flows with road $j$.

As is shown in Figure 2, we choose $\mathrm{K}(\mathrm{K}=4)$ road segments which have the most similar traffic-rate (the rate represents throughput of traffic in a road) evolution for each road segment. Finally, we find out that most neighbors consist of a linear combination of the target road segment. In our solution, we develop this discovery by using an approach: KNN (k-Nearest Neighbors). 


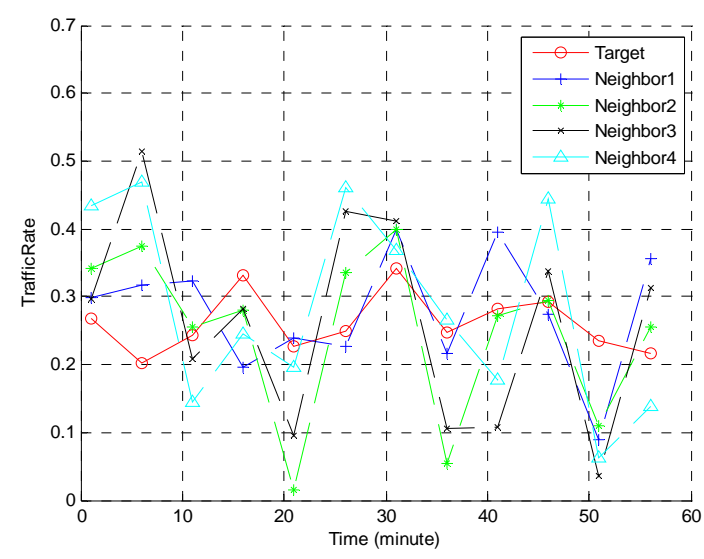

Figure 2. Traffic-rate comparisons between 4 neighbors and target road.

The KNN algorithm to calculate correlation is given as follows:

1) A GPS dataset is used as the train dataset to construct a complete OD matrix.

2) Any row in the OD matrix could be considered as a vector. We take advantage of (1) to calculate similarity of vectors. Always, we set $\mathrm{K}=4$ that a road segment has four neighbors.

3) We use linear regression to find a vector of weights $\omega(k)(k=1,2 \ldots K)$ so that road $i$ is expressed by combination of roadk. In (1), roadi represents the ith row of OD matrix.

$$
\begin{aligned}
\text { Correlation }\left(\operatorname{road}_{i}, \operatorname{road}_{k}\right) & =\cos \theta=\frac{\operatorname{road}_{i} \cdot \text { roadk }_{k}}{\left\|\operatorname{road}_{i}\right\| \times \| \text { road }_{\|}} \\
\operatorname{road}_{i} & =\sum_{k=1}^{K=4} \omega(k) \operatorname{road}_{k}
\end{aligned}
$$

Even though we use just one train dataset to find neighbors for each road segment, the neighbor relations of the road segment can still be hold for other dataset.

\subsection{The Temporal Component}

As is show in the Figure 3, the left picture shows a distribution of traffic-rate from 11:00p.m to 13:00p.m during one day. We interpolate these values and present them in the right picture, which implies that traffic rate changes in a small range (less than 0.1). Thus there is the second important truth: The evolution of traffic volumes on a road segment can keep "stable” during a time interval, which is based on the fact that a number of vehicles do not appear suddenly in the same time at same place, namely $f(i, j, t) \approx f(i, j, t+\Delta t)$.

Meanwhile, from the second fact, we can construct a
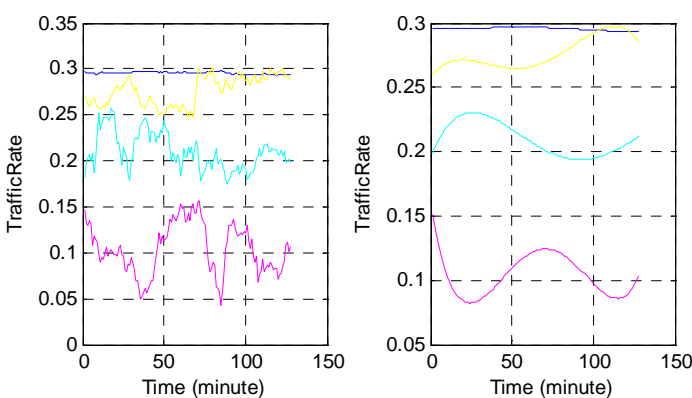

Figure 3. Traffic-rate in 4roads within 2 hours.

temporal constraint matrix TM to show the smoothly changes of traffic volume on each road.

The construction of TM matrix is simple, and the form of TM matrix is given as follows:

$$
T M=\left(\begin{array}{cccc}
1 & -1 & 0 & \ldots \\
0 & 1 & -1 & \ddots \\
0 & 0 & 1 & \ddots \\
\vdots & \ddots & \ddots & \ddots
\end{array}\right)
$$

\subsection{Construction of OD Matrix}

Linear Least Square procedure is often used when constructing OD matrix in industry. Constructing OD matrix is modeled as follows:

$$
Q=B X
$$

Where $Q$ represents total traffic volumes on the road segment and can be easily measured by device like traffic detector [6,7]. In (3), where $B$ is a topological matrix which represents the connectivity of each road segment in an area. Our goal is to find $X$ which is the OD matrix. In practice, $B$ is often a low-rank matrix. Hence, there are probably several solutions to this equation so we have to answer two questions as follows:

1) Which solution is the best?

2) What constraints should we add to estimate $X$ more accurately?

As we mentioned above, $B$ and $X$ are sparse matrices. In implementation, we set a rank value as input and pick the solution whose rank is less than our input as final result.

Through analyzing several experimental results, we are inspired from the spatial component that each road can be linear expressed by its neighbors, so we can take advantage of KNN to build a spatial matrix $S P$ to express which rows in the OD matrix are neighbors.

The construction of SP is given as follows:

1) Use KNN method to find the set of weights for each 
road segment.

2) For $\mathrm{k}=1,2,3, \ldots \mathrm{K}$, we let $S P(i, i)=1$ and $S P\left(i, j_{k}\right)=-\omega(k)$

Then, a spatial constraint equation is given as follows:

$$
S P * X=0
$$

Meanwhile, according to the temporal feature, the temporal constraint equation is given as follows:

$$
T M * X=0
$$

Now, we have acquired two constraint equations through capturing features of traffic volume. In our system, we use standard Linear Least Square (we develop this procedure in Matlab) which is based on (3),(4),(5) to estimate $X$.

\subsection{Real-Time Traffic Condition Description and Alarm}

How to describe traffic condition is a meaningful but difficult problem which always fascinate researchers [9-11], who proposed variety of algorithms to try to predict speed of vehicles. Intuitively, a fast transit velocity implies a good traffic condition. However, another non-negligible is that speed can be influenced by many factors which are difficult to measure, such as behavior of drivers, traffic lights delay and condition of road.

As is shown in Figure 4, we select a taxi randomly and display its changes of speed during an hour. We can see that the taxi stop when $\mathrm{t}=17 \mathrm{~min}$ and runs slow at some time. These are normal phenomenon because of traffic lights which have a significant impact on speed. On the other hand, as is described in Figure 4, speed does not have a regular pattern to predict in reality.

Instead, we propose a solution to capture the traffic status and broadcast warning message to avoid traffic

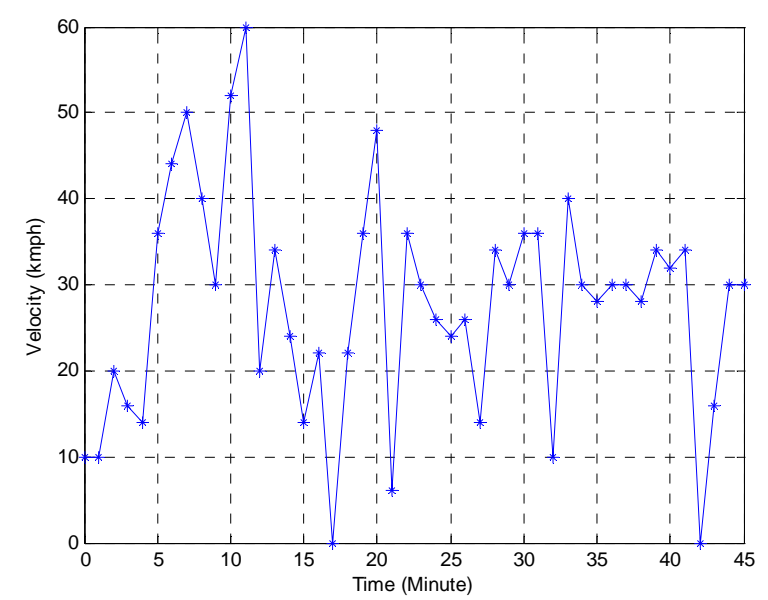

Figure 4. Speed changes during an hour. congestion. We do not just use traffic volumes as a metric to identify traffic status. Because different road has different number of lanes and length, it is unreasonable to use traffic volume to identify traffic jam in such cases.

Considering different condition of roads, in this paper, we define "traffic-rate" $R_{t}$ as a metric to describe current traffic condition. The number of lanes and length of road are taken into account in our design.

We calculate $R_{t}$ using following:

$$
R_{t}=\frac{S_{\text {in }}+1}{S_{\text {in }}+S_{\text {out }}+1}
$$

where $S_{\text {in }}$ expresses the number of vehicles that enter into the road during $[t, t+\Delta t)$, Sout expresses the number of vehicles that leave the road during $[t, t+\Delta t)$.

Meanwhile, we assign a threshold $\lambda$ for each road segment. From a simple perspective, if we find $R_{t}>\lambda$, we think current traffic status is changing. Before we discuss how to classify traffic status in detail, we give a solution to calculate $\lambda$ dynamically.

There is a traditional solution to calculate $\lambda$ : we can analyze the past GPS reports and take advantage of a maximum a posteriori (MAP) classification with both likelihood functions and a priori probabilities to calculate $\lambda$ for each road segment. But this solution cost too much time because of thousands of road segments. More importantly, it does not have enough flexibility.

Now, we propose an experimental formula below to calculate $\lambda$ dynamically.

$$
\lambda=\frac{L \times N}{V(T+D)}
$$

where $V$ expresses the mean speed of vehicles on the road and $\mathrm{T}$ is an estimation value which can be calculated by using GPS information to show how long a taxi may stay in the road. In (7), where $D$ is the traffic light duration, $L$ represents length of the road and $N$ represents number of the lanes. Since on a road, the bigger $L \times N$ is, the more vehicles the road can contain.

However, we observe that traffic light duration can be calculated as follows:

$$
D \approx \frac{L}{V} \times S_{\text {out }}
$$

where $L, V$ and $S_{\text {out }}$ have the same mean we mentioned above. Equation (8) has an intuitive mean that vehicles should leave the road in traffic light duration.

Then, (7) is equivalent to

$$
\lambda=\frac{N}{1+S_{\text {out }}}
$$

Rather than previous studies, we do not simply identify two traffic states: good and bad. In our design, we divide current traffic status into five states: FREE, 
NORMAL, ALERT, BUSY and OVERLOAD. Since we must send warning messages of traffic condition to vehicles before traffic condition becomes worse. Another benefit is that we can make flexible policies when we are processing traffic information. The rules to identify traffic status on a road are given as follows:

1) $0 \leq R_{t}<\lambda$, the traffic state is FREE;

2) $\lambda \leq R_{t}<2 \lambda$, the traffic state is NORMAL;

3) $2 \lambda \leq R_{t}<3 \lambda$, the traffic state is ALERT,

4) $3 \lambda \leq R_{t}<4 \lambda$, the traffic state is BUSY;

5) $4 \lambda \leq R_{t}<1$, the traffic state is OVERLOAD.

In our experiments, $\lambda \approx 0.23$ for most cases. When we detect that current traffic state is ALERT, we start to send warning message to those related vehicles around the road.

\section{Experiments}

\subsection{Experimental Setup}

We have collected a great number of real traffic data which are generated in real time by installing GPS devices in taxis and buses from data center in Shanghai. Even we do not collect all vehicular GPS reports since some vehicles do not install GPS devices, the evaluations also show that our solution can work well.

The fields (id, longitude, latitude, velocity, angle, timestamp) denote the GPS reports, where id identifies a taxi, the pair (longitude, latitude) shows current coordinates of the taxi, timestamp is the time report, the pair(velocity, angle) shows current speed and driving direction of the taxi, which is used with Shanghai map to calculate the short-term destination in our algorithm.

The basic experimental parameters are given as follows:

\subsection{Methodology}

In order to demonstrate the effectiveness of our solution, we have developed a mobility model to generate vehicular GPS reports in a target area. The mobility model consists of three parts:

1) We load GIS map of Shanghai to generate topological matrix.

2) We set source and destination coordinates for each node. Traffic light duration is set according to surveys.

3) The nodes send GPS reports randomly and some nodes do not send. Moreover, for making our simulation close to real life, we also insert real GPS traces into our test dataset.

Our initial OD matrix lost elements at some positions and some elements have error in their value.

At last, we measure performance using the Normalized Mean Absolute Error [4].

$$
N M A E=\frac{\sum_{i, j: M(i, j)=0}|X(i, j)-\hat{X}(i, j)|}{\sum_{i, j: M(i, j)=0}|X(i, j)|}
$$

\subsection{Performance}

Figure 5 shows the performance of our algorithm ST and comparison between SRSVD Base [4], KNN, and ST when GPS reports lost randomly. The default parameters have been listed in Table $\mathbf{1}$. We can clearly realize that ST outperforms the other interpolation algorithms in our experiment. Since we capture spatial and temporal features of traffic flows, ST has more rules and techniques to interpolate missing values than KNN and SRSVD Base. Although there is high GPS data loss, ST can still keep a good performance. From the comparison, we find that there is significant difference between ST and other algorithms when GPS-LOSS-PROBABILITY $=0.6$. KNN works well if enough GPS reports have been collected in the datacenter. However, the performance of KNN decreases sharply when more and more GPS data lost. Because even we have chosen the best $\mathrm{K}$ neighbors for target route, we still do not have another rule to interpolate traffic flows for target road segment.

SRSVD Base does not work well, since it is to some extent a pure technique of matrix calculation. Therefore, it is not able to be adapted to complicated traffic condition.

\subsection{Simulation}

The basic experimental parameters are displayed in Table 1. In followed experiments, we assume that $30 \%$ vehicles do not install GPS devices. Figure 6 shows evolutions of $R_{t}$ on different road segments. At first, we select three different road segments randomly in center of Shanghai. Secondly, we collect the GPS reports on these roads from 11:00 to 12:00 on Jan.7 2007. At last, we recon-

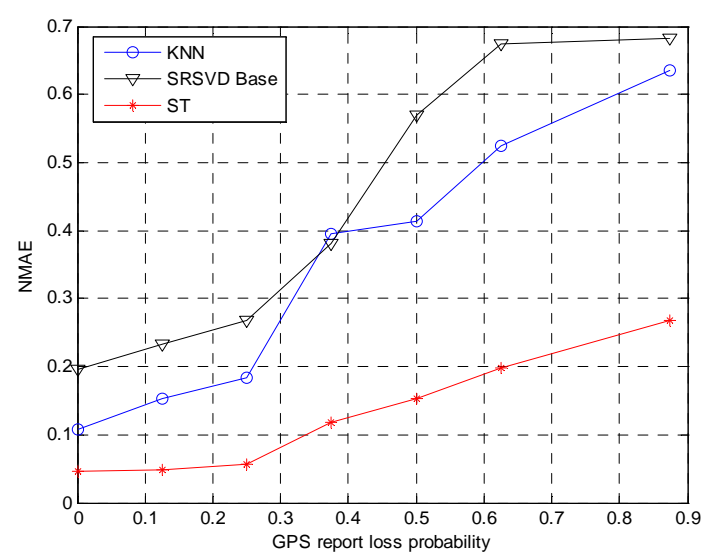

Figure 5. Performance for GPS report loss. 
Table 1: Experimental parameters.

\begin{tabular}{cc}
\hline Target Area & $3.5 \mathrm{~km}^{2}$ \\
\hline Time duration & 2hours (11:00-13:00) \\
Time granularity & $5 \mathrm{~min}$ \\
Number of road segments & 146 \\
Weather & Sunny \\
\hline
\end{tabular}

structed OD matrix using ST to calculate $R_{t}$. In this experiment, we find that traffic lights have little effect on Traffic-Rate and Traffic-Rate changes smoothly.

In order to demonstrate performance of equations of $\lambda$ : We select 10 road segments randomly and monitor the traffic volume from 9:00-22:00 on Jan.10 2007. Then we calculate $R_{t}$ and $\lambda$ every 5 minutes. Meanwhile, we use mean speed of these vehicles to detect current traffic status. Finally, we compare our result with pre-defined traffic state.

Figure 7 shows that the accuracy of our solution and comparison with traditional policy. Apparently, from this experiment, speed is not a good metric to detect traffic condition. Since it is often influenced by many factors, and there are always some vehicles have "strange" driving behaviors because of habits of drivers. On the other side, we can clear see that even $90 \%$ of accuracy can be achieved at sometimes. There are also two least values which are about 0.712 at 12:00 and 0.734 at 17:00. Since they are two important time points when people come off duty, and traffic condition at that time is difficult to be captured. But in general, the performance of threshold-based Traffic-Rate is good and adapted to the real environment.

In Figure 8, the axis of abscissas represents proportion of vehicles that installed GPS devices. This experiment

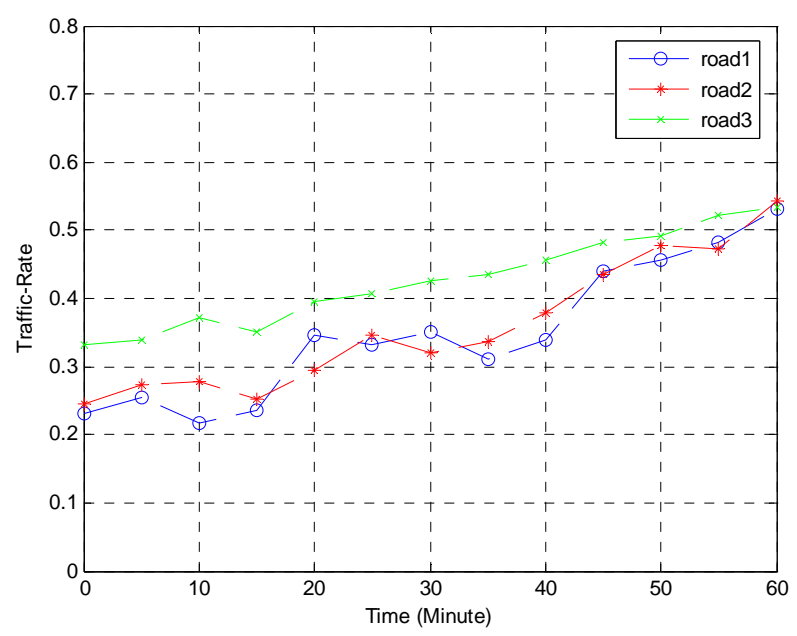

Figure 6. Traffic-Rate changes during an hour on 3 roads.

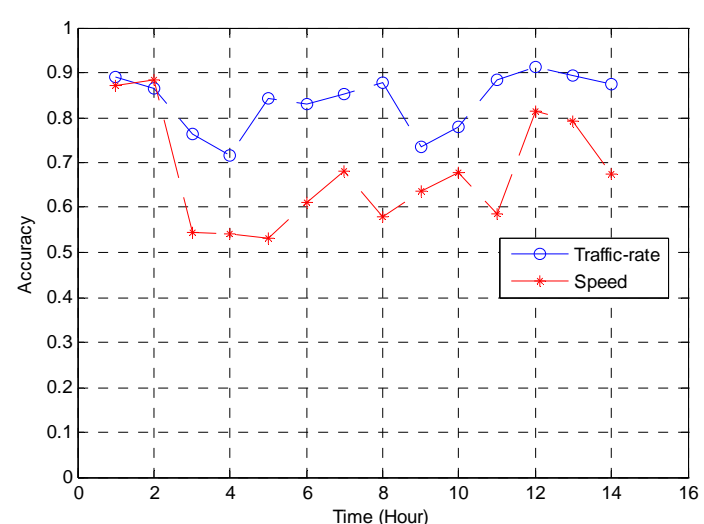

Figure 7. Accuracy of threshold-based Traffic-Rate and mean speed of vehicles.

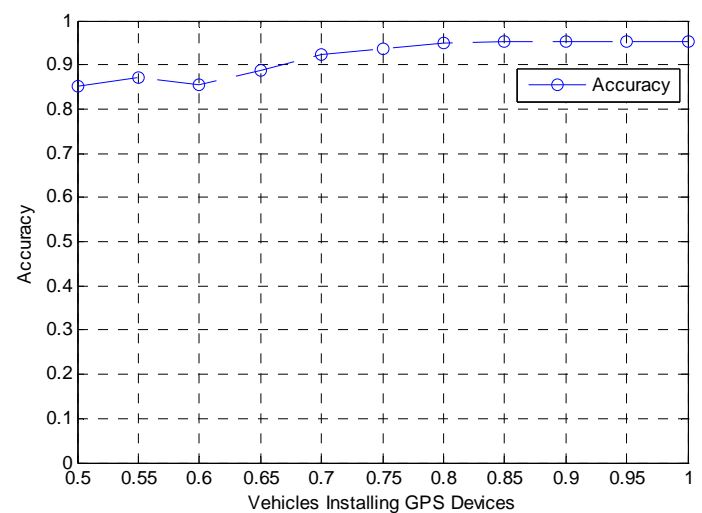

Figure 8. Accuracy of threshold-based Traffic-Rate.

proves that the more vehicles send GPS reports, the better performance of our algorithm is.

\section{Conclusions}

In this paper, we have presented a practical solution to detect traffic condition which is based on OD matrix in an urban city by using a cost-effective system of vehicle GPS sensors. Our algorithm consists of spatial and temporal components which are based on neighbors of roads and stability change of traffic volumes during a time. Meanwhile, we use traffic-rate to capture traffic status rather that speed. As a result, our solution overcomes many limitations in existing approaches and yields a good performance.

\section{References}

[1] D. Bhattacharjee, K. C. Sinha and J. V. Krogmeier, "Modeling the Effects of Traveler Information on Freeway Origin-Destination Demand Prediction,” Transportation Research, Vol. 6, No. 9, 2001, pp. 381-398. 
[2] G. L. Chang and J. F. Wu, "Recursive Estimation of Time-Varying Origin-Destination Flows from Traffic Counts in Freeway Corridors," Transportation Research Part B, Vol. 28, No. 2, 1994, pp. 141-160.

[3] A. Medina, N. Taft, K. Salamatian, S. Bhattacharyya and C. Diet. "Traffic Matrix Estimation: Existing Techniques and New Directions,” Proceedings of ACM SIGCOMM, 2002.

[4] Y. Zhang, M. Roughan, W. Willinger and L. L. Qiu, "Spatio-Temporal Compressive Sensing and Internet Traffic Matrices," Proceedings of ACM SIGCOMM, 2009.

[5] Y. Cho, "Estimating Velocity Fields on a Freeway from Low-Resolution Videos," IEEE Transactions on Intelligent Transportation Systems, Vol.7, No. 4, 2007, pp. 463-469.

[6] W. Lin and C. Daganzo, "A Simple Detection Scheme for Delay-Inducing Freeway Incidents,” Transportation Research, Vol. 31A of Part A, 1997, pp. 141-155.
[7] B. Coifman, "Identifying the Onset of Congestion Rapidly with Existing Traffic Detector,” Transportation Research, Vol. 37 of Part A, 2003, pp. 277-291.

[8] J. Yoon, B. Noble and M. Liu, "Surface Street Traffic Estimation,” Proceedings of ACM Mobicom, 2007

[9] H. Z. Zhu, Y.M. Zhu, M. L., Li and L. M. Ni, "SEER: Metropolitan-Scale Traffic Perception Based on Lossy Sensory Data," Proceedings of ACM INFOCOM, 2009.

[10] M. McNally, J. Marca, C. Rindty and A. Koos. "Tracer: In-Vehicle, Gps-Based, Wireless Technology for Traffic Surveillance and Management,” Technical Report UCBITS-PRR-2003-23, California Partners for Advanced Transit and Highways (PATH), July 2003.

[11] J. Ygnace, C. Drane, Y. Yim and R. Lacvivier. "Travel Time Estimation on the San Francisco Bay Area Network Using Cellular Phones as Probes," Technical Report UCB-ITS-PWB-2000-18, California Partners for Advanced Transit and Highways (PATH), September 2000. 